\title{
Millimeter-wave spectroscopy and modeling of 1,2-butanediol ${ }^{\star}$ Laboratory spectrum in the $59.6-103.6 \mathrm{GHz}$ region
and comparison with the ALMA archived observations
}

\author{
A. Vigorito ${ }^{1}$, C. Calabrese $e^{1,2,3, \star \star}$, S. Melandri ${ }^{1}$, A. Caracciolo ${ }^{1, \star \star \star}$, S. Mariotti ${ }^{4}$, A. Giannetti $^{4}$, \\ M. Massardi ${ }^{4}$, and A. Maris ${ }^{1}$ \\ 1 Dipartimento di Chimica “Giacomo Ciamician”, Università di Bologna, Via Selmi 2, 40126 Bologna, Italy \\ e-mail: assimo.maris@unibo.it \\ 2 Dpto. Química Física, Facultad de Ciencia y Tecnología, Universidad del País Vasco (UPV/EHU), Apartado 644, 48080 Bilbao, \\ Spain \\ 3 Basque Centre for Biophysics (CSIC, UPV/EHU), Bilbao, Spain \\ 4 Istituto di Radioastronomia (IRA-INAF), Via Gobetti 101, 40129 Bologna, Italy
}

Received 24 May 2018 / Accepted 26 July 2018

\section{ABSTRACT}

\begin{abstract}
Context. The continuously enhanced sensitivity of radioastronomical observations allows the detection of increasingly complex organic molecules. These systems often exist in a large number of isomers leading to very congested spectra.

Aims. We explore the conformational space of 1,2-butanediol and provide sets of spectroscopic parameters to facilitate searches for this molecule at millimeter wavelengths.

Methods. We recorded the rotational spectrum of 1,2-butanediol in the 59.6-103.6 GHz frequency region (5.03-2.89 mm) using a free-jet millimeter-wave absorption spectrometer, and we analyzed the properties of 24 isomers with quantum chemical calculations. Selected measured transition lines were then searched on publicly available ALMA Band 3 data on IRAS 16293-2422 B.

Results. We assigned the spectra of six conformers, namely $\mathrm{aG}^{\prime} \mathrm{Ag}, \mathrm{gG}^{\prime} \mathrm{Aa}, \mathrm{g}^{\prime} \mathrm{G}^{\prime} \mathrm{Ag}, \mathrm{aG}^{\prime} \mathrm{G}^{\prime} \mathrm{g}, \mathrm{aG} \mathrm{G}^{\prime} \mathrm{G}$, and g'GAa, to yield the rotational constants and centrifugal distortion constants up to the fourth or sixth order. The most intense signal belong to the aG ${ }^{\prime} \mathrm{Ag}$ species, that is the global minimum. Search for the corresponding $30_{x, 30}-29_{x, 29}$ transition lines toward IRAS $16293-2422$ B was unsuccessful.

Conclusions. Our present data will be helpful for identifying 1,2-butanediol at millimeter wavelengths with radio telescope arrays. Among all possible conformers, first searches should be focused on the $\mathrm{aG}^{\prime} \mathrm{Ag}$ conformers in the $400-800 \mathrm{GHz}$ frequency spectral range.
\end{abstract}

Key words. molecular data - line: identification - methods: laboratory: molecular - methods: data analysis techniques: spectroscopic - radio lines: ISM

\section{Introduction}

Millimeter and submillimeter astronomy is a fundamental technique for searching complex organic molecules (COMs) in circumstellar envelopes and interstellar medium (Brünken \& Schlemmer 2015). However laboratory spectra acquisition, assignment, and analysis are necessary for the correct identification of the features contained in ongoing spectral surveys.

Among all the detected molecules, diols (organic compounds with two hydroxyl groups) are an object of chemical interest because of their similarity with important biological building block molecules such as sugar alcohols. The prototype of these species, ethylene glycol, is one of the largest COMs detected in space so far, as attested by the Cologne Database for Molecular

\footnotetext{
* The measured and predicted transition lines for six conformers of 1,2-butanediol are only available at the CDS via anonymous ftp to cdsarc.u-strasbg. fr (130.79.128.5) or via http://cdsarc. u-strasbg.fr/viz-bin/qcat?]/A+A/619/A140

$\star \star$ Co-first author.

$\star \star \star$ Present address: Dipartimento di Chimica, Biologia e Biotecnologie, Università di Perugia, 06123 Perugia, Italy.
}

Spectroscopy (CDMS 2001; Müller et al. 2001, 2005). Lines attributable to the most stable conformer of ethylene glycol were detected in a variety of astronomical environments, such as the Galactic center, hot cores, hot corinos, and comets (Hollis et al. 2002; Requena-Torres et al. 2008; Maury et al. 2014; Coutens et al. 2015; Jørgensen et al. 2016; Crovisier et al. 2004; Biver et al. 2014; Fuente et al. 2014; Brouillet et al. 2015), and tentatively, toward the two high-mass star-forming regions W51/e2 (Kalenskii \& Johansson 2010; Lykke et al. 2015) and G34.3+0.2 (Lykke et al. 2015).

It is worth noting that recently a second conformer of ethylene glycol, lying at about $2.5 \mathrm{~kJ} \mathrm{~mol}^{-1}$ above the lowest energy form (Müller \& Christen 2004), has also been observed both toward IRAS 16293-2422 (Jørgensen et al. 2016) and the Orion Kleinmann-Low nebula (Favre et al. 2017).

Regarding larger diols, observation searches for 1,2propanediol (Lovas et al. 2009) and 1,3-propanediol (Plusquellic et al. 2009) toward Sgr B2 (N-LMH) were made as part of the Green Bank Telescope (GBT) Prebiotic Interstellar Molecule Survey Legacy Project (PRIMOS 2013; Remijan et al. 2013), but no transitions were detected beyond the $1 \sigma$ root mean square noise limit. 
The decrease of column densities of molecules with increasing molecular weight is one of the main effects that hinder the detection of larger diols in interstellar space. Nevertheless, thanks to the enhanced sensitivity of the latest radioastronomy observations, their future detection cannot be excluded. On the other hand, the increasing size of the alkyl chain confers a molecular flexibility that is often characterized by a great conformational complexity and is not easy to solve.

In this context, we report a detailed conformational analysis and the laboratory millimeter spectrum of 1,2-butanediol (12BD, hereafter), the ethyl substituted form of ethylene glycol. The high level of flexibility of 12BD requests a nontrivial conformational study (see Sect. 2) in order to understand which are the more abundant species or which provide the most intense spectroscopic signals. The laboratory extraction and modeling of the rotational spectrum are described in Sects. 3 and 4 and the resulting prediction are described in Sect. 5. Finally in Sect. 6, the laboratory investigation has been followed by a tentative search in astronomical observation data extracted from the Atacama Large Millimeter/submillimeter Array (ALMA) Science Archive.

\section{Conformational analysis}

Except for 12BD, rotational spectroscopy investigations are reported for all linear diols containing up to four carbon atoms as follows: ethylene glycol or 1,2-ethanediol (12ED; Marstokk \& Møllendal 1974; Walder et al. 1980; Christen et al. 1995, 2001; Christen \& Müller 2003; Müller \& Christen 2004), 1,2-propanediol (12PD; Caminati 1981; Lockley et al. 2002; Lovas et al. 2009; Bossa et al. 2014), 1,3-propanediol (13PD; Caminati et al. 1995; Plusquellic et al. 2009), 1,3-butanediol (13BD; Caminati \& Corbelli 1982; Velino et al. 2011), 2,3butanediol (23BD; Paul et al. 2007), and 1,4-butanediol (14BD; Evangelisti et al. 2013).

Even though diols may exist in several distinct conformations, the more stable structures are those stabilized by the intramolecular hydrogen bond between the two hydroxyl groups $(\mathrm{OH} \cdots \mathrm{OH})$. Actually, only this family of conformers was detected by laboratory rotational spectroscopy.

Concerning diols with hydroxyl groups at the outer carbon atoms such as 12ED, 13PD, and 14BD (Fig. 1, first row), two nonequivalent structures were observed, each of these corresponding to four equivalent conformers. The rotational spectra are complicated by a strong rotation-tunneling interaction depending on the interconversion energy barriers between the equivalent species.

In contrast, in the cases of 12PD, 12BD, and 13BD (Fig. 1, second row) the conformers are not equivalent because of the presence of an outer methyl group. Seven and five species were observed for 12PD and 13BD, respectively. It can be also pointed out that in these species the carbon atom binding four different substituents is a chiral center giving rise to the specular and isoenergetic $R$ and $S$ configurations. However, these configurations can be considered spectroscopically equivalent in conventional rotational experiments.

In 23BD (Fig. 1, third row), because of the presence of two chiral carbon atoms, four isomers exist; these are $(R, R)$ and specular $(S, S),(R, S)$ and specular $(S, R)$. Among the possible conformations, only one species was observed for each pair, and a tunneling motion due to the interchange of the hydrogen bonded hydroxyl groups was observed for $(R, R / S, S)$.

The molecular structure of $12 \mathrm{BD}$ is defined by the HO-CC hydroxyl dihedral angles ( $\tau_{1}$ and $\tau_{4}$, Fig. 1) and the OC-CO and CC-CC skeletal torsional angles ( $\tau_{2}$ and $\tau_{3}$, respectively). The

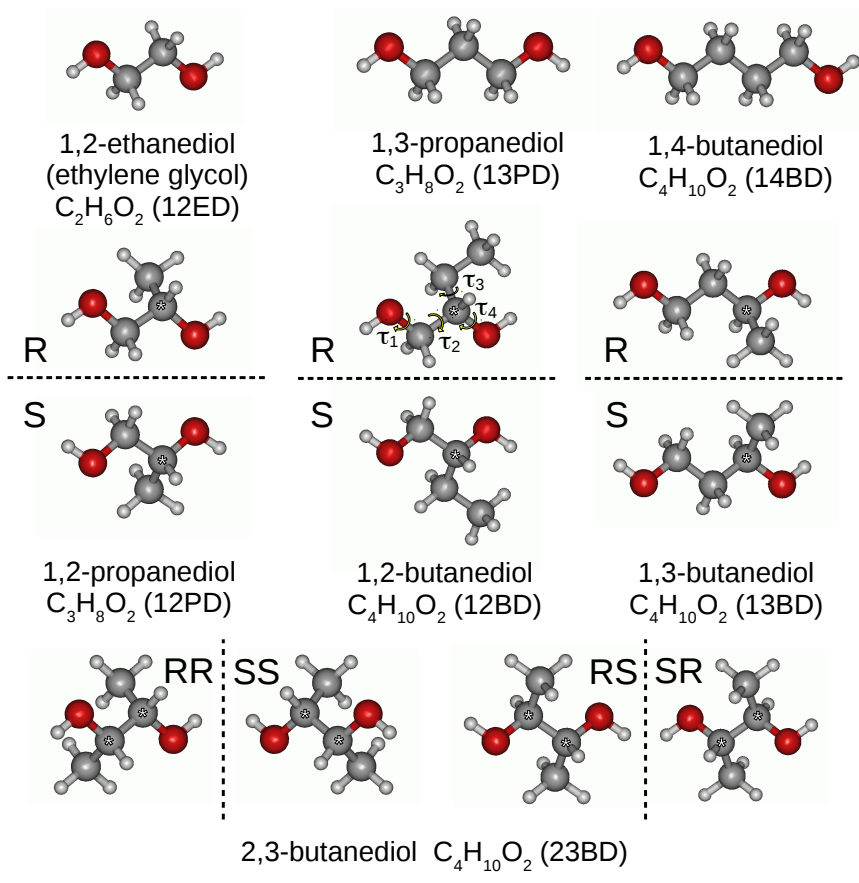

Fig. 1. Diols up to 4 carbon atoms. Gray, white, and red spheres represent the carbon $(\mathrm{C})$, hydrogen $(\mathrm{H})$, and oxygen $(\mathrm{O})$ atoms, respectively. Asterisks indicate chiral atoms. The dashed line indicates the mirror plane between the chiral forms. The labeling of dihedral angles used in the text is shown.

methyl group internal rotation is not relevant because it leads to equivalent minima. Because of steric hindrance, for each $\tau_{i}$ three staggered orientations are possible, giving rise to $3^{4}=81$ possible nonequivalent conformers. The labeling chosen for the identification of these conformers reflects the $\tau_{1}, \tau_{2}, \tau_{3}$, and $\tau_{4}$ values, respectively, using a sequence of the four letters $\mathrm{xXY}$ $\left(X, Y=A, G, G^{\prime} ; x, y=a, g, g^{\prime}\right.$ ). These letters indicate the three staggered positions of the four dihedral angles as follows: anti $\left(\tau \sim 180^{\circ}\right)$, gauche $\left(\tau \sim 60^{\circ}\right)$, or gauche' $\left(\tau \sim-60^{\circ}\right)$. Capital and lower case letters refer to the backbone and hydroxyl torsions, respectively.

As regards the chirality, to simplify the discussion, we refer only to the $R$ configuration. The corresponding $S$ configuration is achieved changing the sign of all dihedral angles.

Assuming that 12BD behaves like the already studied diols, the most stable forms are characterized by the hydrogen bond between the hydroxyl groups. This kind of interaction can take place in 24 of the 81 possible conformers as follows: $\mathrm{aGYg}^{\prime}$, gGYg ${ }^{\prime}, a G Y g^{\prime}, g G Y g^{\prime}, g^{\prime} G^{\prime} Y g, a G^{\prime} Y g, g G^{\prime} Y a$, and gG' $\mathrm{Yg}^{\prime}$ with $\mathrm{Y}=\mathrm{A}, \mathrm{G}, \mathrm{G}^{\prime}$. Their structures were optimized at the B3LYPD3/aug-cc-pVTZ level of calculation using the Gaussian 09 quantum chemistry package (Frisch et al. 2013) and subsequent harmonic vibrational frequency calculations confirmed that the optimized geometries correspond to real minima in the conformational potential energy surface.

The B3LYP density functional is one of the most widely used for the quantum mechanical prediction of molecular properties. Including the empirical dispersion correction (D3; Grimme et al. 2010), and using a very extended basis set (aug-cc-pVTZ) should ensure reliable modeling of the intramolecular hydrogen bond interaction and, consequently, an accurate description of the relative energies and structures of the conformers.

The 24 hydrogen-bonded conformers, shown in Fig. 2 with their zero-point corrected relative energies, were considered in 


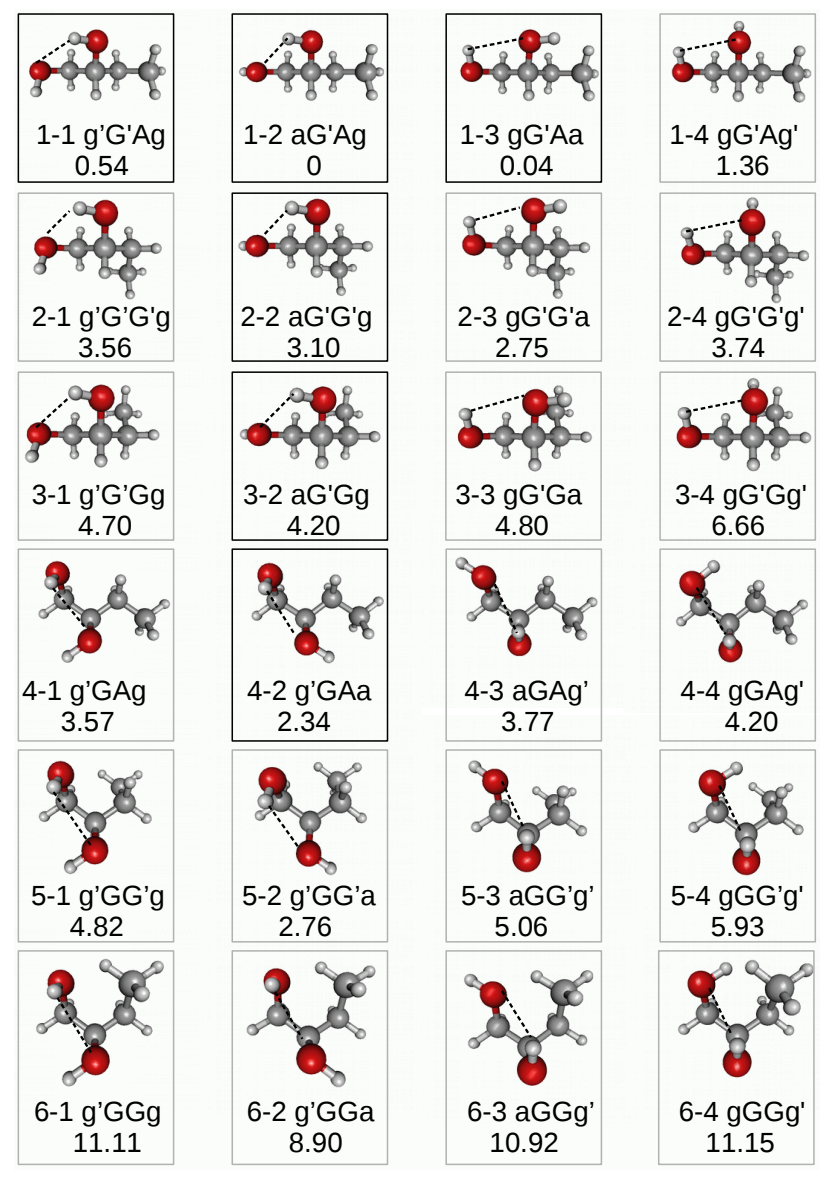

Fig. 2. Theoretical (B3LYP-D3/aug-cc-pVTZ) structures and zero-point corrected relative electronic energy values $\left(\Delta E_{0} / \mathrm{kJ} \mathrm{mol}^{-1}\right)$ of the 24 hydrogen-bonded conformers of $R-12 \mathrm{BD}$. Gray, white, and red spheres represent the carbon $(\mathrm{C})$, hydrogen $(\mathrm{H})$ and oxygen $(\mathrm{O})$ atoms, respectively. Black frames indicate the observed species.

the analysis of the rotational spectrum. For the sake of clarity, besides the label, a numerical code is also introduced. It is composed of two digits, referring to the skeletal and hydroxyl groups configurations, respectively.

The theoretical relative energy values of 20 conformers lie below $7 \mathrm{~kJ} \mathrm{~mol}^{-1}$, suggesting that at room temperature the conformational population is spread on a large number of species. This behavior, common in flexible molecules, complicates both the detection and analysis of the spectra. In the present work, such a difficulty has been overcome exploiting the rotational and conformational cooling produced by a supersonic expansion of the sample seeded in monoatomic gases.

\section{Experiment}

12BD (purity 98\%, molecular weight $90.121 \mathrm{~g} \mathrm{~mol}^{-1}$ ) was purchased from Sigma-Aldrich and used without any further purification. It appears as a colorless, viscous liquid at ambient conditions. The melting point is $-50{ }^{\circ} \mathrm{C}(223 \mathrm{~K})$ and the boiling point is $191-192^{\circ} \mathrm{C}(464-465 \mathrm{~K})$. Both argon and helium, purchased from SIAD (Società Italiana Acetilene e Derivati), were used as carrier gases.

The sample was heated to about $80^{\circ} \mathrm{C}(353 \mathrm{~K})$ and a stream of the carrier gas $\left(P_{0}(\mathrm{Ar})=20 \mathrm{kPa}, P_{0}(\mathrm{He})=40 \mathrm{kPa}\right)$ was flowed over the sample and then expanded to about $P_{b}=0.5 \mathrm{~Pa}$ through a heated $0.3 \mathrm{~mm}$ diameter pinhole nozzle. The data reported by
Steele et al. (1996) and Verevkin (2004) were used to extrapolate the $12 \mathrm{BD}$ vapour pressure at the working temperature: $0.545 \mathrm{kPa}$ at $353 \mathrm{~K}$. In this way the concentration of $12 \mathrm{BD}$ was estimated to be around $2.7 \%$ and $1.4 \%$ in argon and helium, respectively.

Rotational spectra in the millimeter-wave region (5.03$2.89 \mathrm{~mm}, 59.6-103.6 \mathrm{GHz})$ were recorded using a Starkmodulated free-jet absorption spectrometer. The spectrometer has a resolution of about $300 \mathrm{kHz}$ and an estimated accuracy of about $50 \mathrm{kHz}$. With respect to the ordinary experimental setup described in Melandri et al. (1995, 1996) and Calabrese et al. (2015), some modifications on the multipliers chain were performed to obtain frequencies in the $W$ band, more precisely, the same $K$-band frequency generator equipment was driving a $\times 4$ frequency multiplier, followed by a dial attenuator. The lens limitation over $73 \mathrm{GHz}$ and the over-moded driven waveguide was systematically checked and no power loss was attested up to $103 \mathrm{GHz}$.

\section{Rotational spectrum of 1,2-butanediol}

To acquire an overview of the 12BD rotational spectrum, two fast scans covering the $59.6-74.4 \mathrm{GHz}$ frequency range were run using both helium and argon as carrier gas. The spectrum recorded with helium shows a much larger number of lines with respect to the spectrum recorded in argon, suggesting that the population of the sample is constituted by several conformers and that collisional cooling favors the overcoming of the conformational potential energy barriers, giving rise to effective relaxation processes in the expansion in argon.

Predictions based on the theoretical rotational constants and electric dipole moment components reported in Table 1, allowed for the assignment of the most intense spectral lines of six different species. Then, substituting the predictions with the experimental transitions, other frequency assignments could be made until the signal-to-noise ratio was too low to measure with reasonable certainty. In this iterative process, the need for centrifugal distortion constants was checked and they were inserted as long as substantial reduction of the rms error was obtained and until their values could be determined with sufficient precision.

The assigned frequencies and corresponding rotational quantum numbers are available in the supplementary material, as outlined in the appendix. For all conformers, a distribution plot of the upper state $J$ and $K_{a}$ values of all measured transitions included in the least-squares fit is shown in Fig. 3. The CALPGM suite of programs (Pickett 1991) was used to fit the data and predict the rotational spectra, applying Watson's $S$ reduction and the $I^{\mathrm{r}}$ representation of the rotational Hamiltonian (Watson 1977).

The final sets of spectroscopic parameters (rotational and centrifugal distortion constants) are reported in Table 2 with a rough indication of the intensity ratio of the $\mu_{b}$-type to $\mu_{c}$-type transitions, which form a quartet of lines.

Based on the different types of rotational transitions observed for each species, theoretical relative energies, and comparison between experimental and theoretical spectroscopic constants, the assigned spectra could be uniquely correlated to the conformers $\mathrm{aG}^{\prime} \mathrm{Ag}, \mathrm{g}^{\prime} \mathrm{G}^{\prime} \mathrm{Ag}, \mathrm{gG} \mathrm{G}^{\prime} \mathrm{Aa}, \mathrm{aG}^{\prime} \mathrm{Gg}, \mathrm{aG}^{\prime} \mathrm{G}^{\prime} \mathrm{g}$, and $\mathrm{g}^{\prime} \mathrm{GAa}$.

The most intense spectral lines, both in helium and argon expansions, belong to the $R$-branch $\mu_{b}$ and $\mu_{c}$ degenerate transitions of conformer $\mathrm{aG}^{\prime} \mathrm{Ag}$, indicating that it is the global minimum. This is in agreement both with the quantum mechanical results and experimental findings on related molecules 12ED and 12PD, for which the global minima have the same heavy atoms arrangement $\left(\mathrm{aG}^{\prime} \mathrm{g}\right)$. 
A\&A 619, A140 (2018)

Table 1. Theoretical results for the 24 hydrogen bonded conformers of 1,2-butanediol, obtained at the B3LYP-D3/aug-cc-pVTZ level of calculation.

\begin{tabular}{|c|c|c|c|c|c|c|c|c|c|c|c|c|}
\hline & Conf. & $\begin{array}{c}E_{\mathrm{e}} \\
\text { Hartree }\end{array}$ & $\begin{array}{c}\Delta E_{\mathrm{e}} \\
\mathrm{kJ} \mathrm{mol}^{-1}\end{array}$ & $\begin{array}{c}E_{0} \\
\text { Hartree }\end{array}$ & $\begin{array}{c}\Delta E_{0} \\
\mathrm{~kJ} \mathrm{~mol}^{-1}\end{array}$ & $\begin{array}{c}A_{\mathrm{e}} \\
\mathrm{MHz}\end{array}$ & $\begin{array}{c}B_{\mathrm{e}} \\
\mathrm{MHz}\end{array}$ & $\begin{array}{c}C_{\mathrm{e}} \\
\mathrm{MHz}\end{array}$ & $\begin{array}{r}\mu_{a} \\
\mathrm{D}\end{array}$ & $\begin{array}{r}\mu_{b} \\
\mathrm{D}\end{array}$ & $\begin{array}{r}\mu_{c} \\
\mathrm{D}\end{array}$ & $\begin{array}{l}\mu \\
\mathrm{D}\end{array}$ \\
\hline $1-1$ & $\mathrm{~g}^{\prime} \mathrm{G}^{\prime} \mathrm{Ag}$ & -309.028967 & 0.22 & -308.887333 & 0.54 & 7812.70 & 1921.23 & 1671.84 & -0.21 & 1.68 & -1.44 & 2.22 \\
\hline $1-2$ & $\mathrm{aG}^{\prime} \mathrm{Ag}$ & -309.029044 & 0.01 & -308.887539 & 0.00 & 7811.31 & 1937.00 & 1681.80 & 1.28 & 1.85 & -0.44 & 2.29 \\
\hline $1-3$ & $\mathrm{gG}^{\prime} \mathrm{Aa}$ & -309.029049 & 0.00 & -308.887525 & 0.04 & 7673.24 & 1929.04 & 1666.98 & -2.59 & 0.19 & -0.36 & 2.62 \\
\hline $1-4$ & $\mathrm{gG}^{\prime} \mathrm{Ag}^{\prime}$ & -309.028531 & 1.36 & -308.886956 & 1.53 & 7575.92 & 1928.69 & 1670.44 & -2.00 & -0.56 & 1.44 & 2.53 \\
\hline $2-1$ & $\mathrm{~g}^{\prime} \mathrm{G}^{\prime} \mathrm{G}^{\prime} \mathrm{g}$ & -309.027943 & 2.90 & -308.886183 & 3.56 & 5462.10 & 2246.60 & 1722.97 & 0.72 & 1.87 & -1.17 & 2.33 \\
\hline $2-2$ & $\mathrm{aG}^{\prime} \mathrm{G}^{\prime} \mathrm{g}$ & -309.028000 & 2.75 & -308.886357 & 3.10 & 5473.98 & 2266.50 & 1730.92 & 0.17 & 2.27 & -0.71 & 2.38 \\
\hline $2-3$ & $\mathrm{gG}^{\prime} \mathrm{G}^{\prime} \mathrm{a}$ & -309.028166 & 2.32 & -308.886490 & 2.75 & 5380.29 & 2270.34 & 1723.68 & -2.33 & 1.29 & -0.46 & 2.71 \\
\hline $2-4$ & $\mathrm{gG}^{\prime} \mathrm{G}^{\prime} \mathrm{g}^{\prime}$ & -309.027882 & 3.06 & -308.886114 & 3.74 & 5339.64 & 2275.19 & 1725.25 & -2.17 & 0.55 & 1.38 & 2.63 \\
\hline $3-1$ & $\mathrm{~g}^{\prime} \mathrm{G}^{\prime} \mathrm{Gg}$ & -309.027612 & 3.77 & -308.885747 & 4.70 & 5943.35 & 2179.45 & 1916.68 & -0.31 & 1.24 & -1.72 & 2.15 \\
\hline $3-2$ & $\mathrm{aG}^{\prime} \mathrm{Gg}$ & -309.027670 & 3.62 & -308.885939 & 4.20 & 5989.98 & 2190.10 & 1918.80 & -0.75 & 2.16 & 0.20 & 2.30 \\
\hline $3-3$ & $\mathrm{gG}^{\prime} \mathrm{Ga}$ & -309.027468 & 4.15 & -308.885711 & 4.80 & 5860.98 & 2187.51 & 1905.08 & -2.19 & 0.90 & -0.86 & 2.52 \\
\hline $3-4$ & $\mathrm{gG}^{\prime} \mathrm{Gg}^{\prime}$ & -309.026817 & 5.86 & -308.885003 & 6.66 & 5857.83 & 2172.14 & 1893.94 & -2.50 & -0.49 & 0.63 & 2.62 \\
\hline $4-1$ & $\mathrm{~g}^{\prime} \mathrm{GAg}$ & -309.027808 & 3.26 & -308.886180 & 3.57 & 5923.82 & 2207.55 & 1929.32 & -0.52 & -1.19 & 1.71 & 2.15 \\
\hline $4-2$ & $\mathrm{~g}^{\prime} \mathrm{GAa}$ & -309.028170 & 2.31 & -308.886649 & 2.34 & 5910.35 & 2207.26 & 1926.18 & -2.25 & -0.48 & 0.83 & 2.44 \\
\hline $4-3$ & aGAg' & -309.027642 & 3.69 & -308.886103 & 3.77 & 5944.60 & 2210.23 & 1936.49 & 1.71 & -1.42 & -0.79 & 2.36 \\
\hline $4-4$ & $\mathrm{gGAg}^{\prime}$ & -309.027706 & 3.52 & -308.885939 & 4.20 & 5968.54 & 2181.83 & 1912.71 & 0.32 & -2.32 & 0.35 & 2.37 \\
\hline $5-1$ & $\mathrm{~g}^{\prime} \mathrm{GG}^{\prime} \mathrm{g}$ & -309.027548 & 3.94 & -308.885701 & 4.82 & 4289.99 & 2766.95 & 2038.48 & -0.10 & -1.07 & 1.72 & 2.03 \\
\hline $5-2$ & $\mathrm{~g}^{\prime} \mathrm{GG}^{\prime} \mathrm{a}$ & -309.028261 & 2.07 & -308.886489 & 2.76 & 4344.00 & 2735.29 & 2027.60 & -1.61 & -1.64 & 0.38 & 2.34 \\
\hline $5-3$ & $\mathrm{aGG}^{\prime} \mathrm{g}^{\prime}$ & -309.027233 & 4.77 & -308.885610 & 5.06 & 4333.14 & 2736.56 & 2033.05 & -0.18 & -2.17 & 0.72 & 2.29 \\
\hline $5-4$ & $\mathrm{gGG}^{\prime} \mathrm{g}^{\prime}$ & -309.027064 & 5.21 & -308.885278 & 5.93 & 4342.28 & 2696.48 & 2008.62 & 1.85 & -1.70 & -0.07 & 2.51 \\
\hline $6-1$ & $\mathrm{~g}^{\prime} \mathrm{GGg}$ & -309.025186 & 10.14 & -308.883306 & 11.11 & 4147.98 & 2944.79 & 2376.10 & -0.24 & -1.89 & -1.05 & 2.18 \\
\hline $6-2$ & $\mathrm{~g}^{\prime} \mathrm{GGa}$ & -309.025791 & 8.55 & -308.884149 & 8.90 & 4139.47 & 2955.26 & 2368.80 & 1.68 & -1.59 & -0.73 & 2.43 \\
\hline $6-3$ & aGGg' & -309.025068 & 10.45 & -308.883380 & 10.92 & 4166.68 & 2925.26 & 2365.31 & 1.47 & -1.43 & -1.33 & 2.45 \\
\hline $6-4$ & aGGg' & -309.025192 & 10.12 & -308.883293 & 11.15 & 4181.25 & 2915.52 & 2343.73 & 1.07 & 1.68 & -1.37 & 2.42 \\
\hline
\end{tabular}

Notes. Columns are: electronic energy values and corresponding zero-point vibration corrected values, equilibrium rotational constants, and electric dipole moment components.

Weaker lines were then assigned to the $\mathrm{aG}^{\prime} \mathrm{Gg}, \mathrm{aG}^{\prime} \mathrm{G}^{\prime} \mathrm{g}$ conformers, which differ for the orientation of the alkyl tail $\left(\tau_{3}\right)$ and the $\mathrm{g}^{\prime} \mathrm{GAa}$ conformer, which presents a different OCCO orientation $\left(\tau_{2}\right)$. This latter conformation can be considered the homologue of $\mathrm{g}^{\prime} \mathrm{Ga}-12 \mathrm{ED}$ and $\mathrm{g}^{\prime} \mathrm{Ga}-12 \mathrm{PD}$. The lines belonging to these three less populated conformers appear slightly depleted in argon expansion, indicating that a partial relaxation to the global minimum takes place.

As remarked above, the rotational spectrum recorded using helium as carrier gas, showed additional lines that were assigned to the $\mathrm{g}^{\prime} \mathrm{G}^{\prime} \mathrm{Ag}$ and $\mathrm{gG}^{\prime} \mathrm{Aa}$ conformers. A clear example of this is reported in Fig. 4, where a portion of the spectra collected using helium and argon are compared. In this frequency region lie the $K_{a}=6 \leftarrow 5 Q$-branch transitions of both the global $\left(\mathrm{aG}^{\prime} \mathrm{Ag}\right)$ and local ( $\mathrm{g}^{\prime} \mathrm{G}^{\prime} \mathrm{Ag}$ ) minima and the $Q$ branch of the less stable conformer $\left(\mathrm{g}^{\prime} \mathrm{G}^{\prime} \mathrm{Ag}\right)$ appears absolutely depleted in the argon spectra. The reason for this is that, considering similar expansion velocities for both carrier gases, the higher mass of argon produces a greater kinetic energy, which entails at the ability to overcome the conformational barriers.

Since the $\mathrm{g}^{\prime} \mathrm{G}^{\prime} \mathrm{Ag}$ and $\mathrm{gG}^{\prime} \mathrm{Aa}$ conformers differ from the global minimum only for the orientation of the hydroxyl group, the depletion of their lines in argon expansion can be ascribed to a strong relaxation process along the hydroxyl internal rotation path. According to Ruoff et al. (1990) the upper limit of the corresponding barriers is $4-5 \mathrm{~kJ} \mathrm{~mol}^{-1}$. It is worth noting that the hydrogen bond is maintained both in the $\mathrm{g}^{\prime} \mathrm{G}^{\prime} \mathrm{Ag} \rightarrow \mathrm{aG}^{\prime} \mathrm{Ag}$ and $\mathrm{gG}^{\prime} \mathrm{Aa} \rightarrow \mathrm{aG}^{\prime} \mathrm{Ag}$ isomerization processes.

Relative intensity measurements on the observed $Q$-branch bands match with a rotational temperature of $3 \mathrm{~K}$ in argon expansion and $5 \mathrm{~K}$ when using helium as carrier gas, confirming the greater cooling efficiency of argon with respect to helium.
The line intensity ratio of $\mathrm{g}^{\prime} \mathrm{G}^{\prime} \mathrm{Ag}$ to $\mathrm{aG}^{\prime} \mathrm{Ag}$ conformers is about 1:3. This value, weighted on the theoretical $\mu_{b}{ }^{2}+\mu_{c}{ }^{2}$ values (Table 1), leads to a $\mathrm{g}^{\prime} \mathrm{G}^{\prime} \mathrm{Ag}: \mathrm{aG}^{\prime} \mathrm{Ag}=1: 4$ population ratio. Assuming that this population ratio is not modified by the expansion, and it is the ratio established at the pre-expansion equilibrium temperature, the corresponding relative energy is $3.9 \mathrm{~kJ} \mathrm{~mol}^{-1}$.

Regarding the $\mathrm{gG}^{\prime} \mathrm{Aa}$ conformer, the intensity of the $\mu_{a^{-}}$ type transition lines are similar to those of $\mathrm{aG}^{\prime} \mathrm{Ag}$. Unfortunately, owing to an incomplete Stark-modulation of the spectral lines, it is not possible to determine a reliable intensity ratio. Nevertheless, considering that the $\mu_{a}$ ratio is about 2 , we can state that the $\mathrm{gG}^{\prime} \mathrm{Aa}$ conformer is less populated than the $\mathrm{aG}^{\prime} \mathrm{Ag}$ conformer.

Finally, no internal rotation splittings due to the terminal methyl group were resolved in the spectra. The predictions based on the experimental rotational constants and theoretical geometries indicate that, in the investigated spectral region, these splittings could be appreciable mainly on some $Q$-type transitions, expecting a separation of $0.4 \mathrm{MHz}$ between the lines. Since this value lies within the resolution limit of our spectrometer, the measured transitions appear affected in the $\mathrm{aG}^{\prime} \mathrm{Ag}$ and $\mathrm{g}^{\prime} \mathrm{G}^{\prime} \mathrm{Ag}$ fits by a high deviation.

\section{Prediction of the rotational spectrum}

To provide useful data for the detection of $12 \mathrm{BD}$ in space, the rotational spectra of the six observed conformers were predicted. The results, based on the experimental spectroscopic constants (Table 2) and theoretical electric dipole moment components (Table 1), are given in the supplementary material, as outlined in the appendix. The prediction sample comprises lines that 

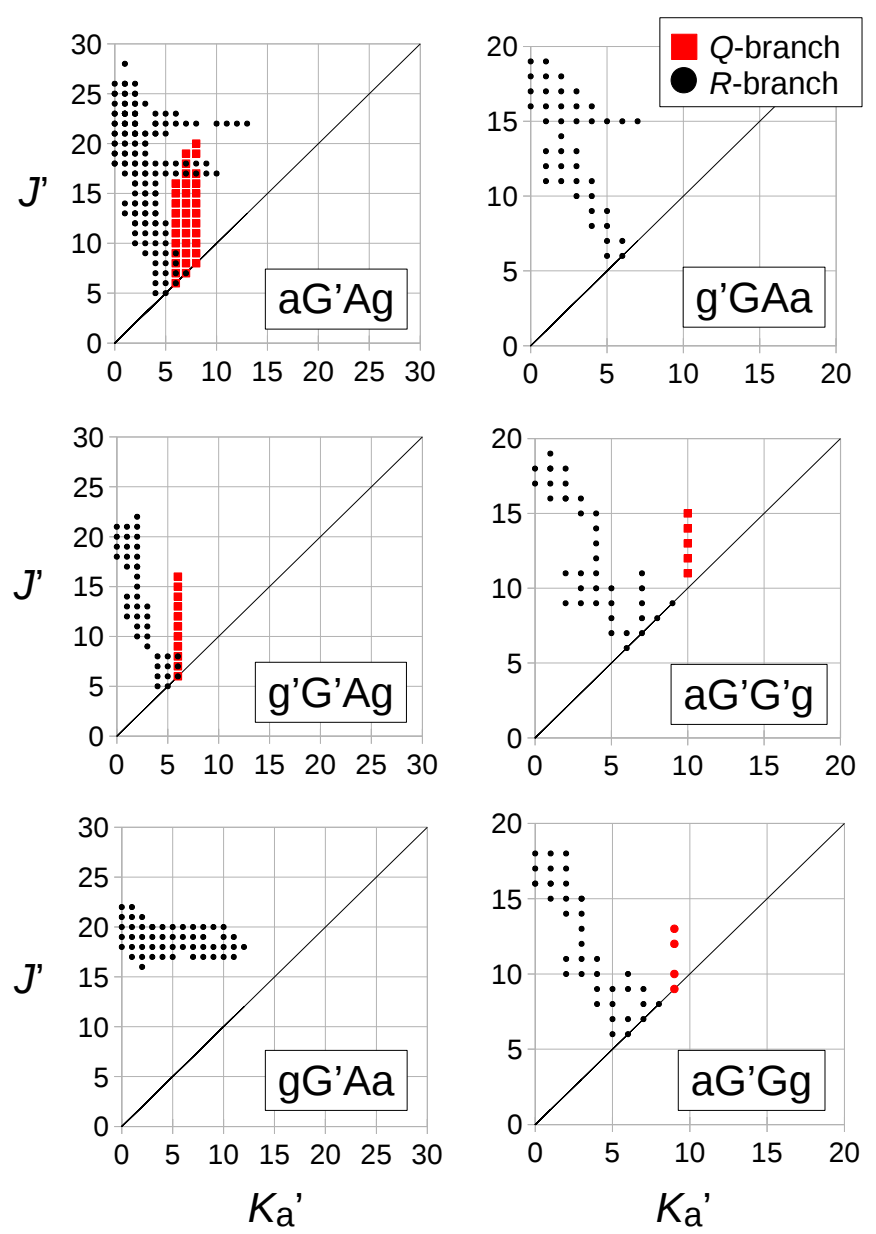

Fig. 3. Data set distribution plots of $J$ ' and $K^{\prime}{ }_{a}$ of all measured transitions for the spectrum of 1,2-butanediol.

have upper state energy $E_{\mathrm{up}}<100 \mathrm{~K}$, and Einstein's coefficient $A>10^{-7} \mathrm{~s}^{-1}$ evaluated as $A=\left(16 \pi^{3} v^{3}\right) /\left(3 \epsilon_{0} \mathrm{hc}^{3}\right) S_{i j} \mu_{\mathrm{g}}^{2}$.

The overall shape of the predicted spontaneous emission probability of $\mathrm{aG}^{\prime} \mathrm{Ag}-12 \mathrm{BD}$ up to $1 \mathrm{THz}$ is illustrated on the top of Fig. 5. The maximum emission is expected between 500 and $800 \mathrm{GHz}$. In Fig. 5 also the predicted absorption spectra at 5, 75 , and $300 \mathrm{~K}$ are compared. At these temperatures, the maximum absorption intensities lie in the 50-100, 200-400, and 400$700 \mathrm{GHz}$ frequency ranges, respectively. The partition function of the six observed conformers of 12BD in the 5-300 K temperature range, given in Table 3 , allows for the evaluation of the absorption coefficient.

\section{Search on ALMA data archives}

IRAS 16293-2422 is a well-studied Class 0 protostar consisting of two main sources denoted as components $\mathrm{A}$ and $\mathrm{B}$. This source is a prototypical hot corino and it therefore has a rich molecular line spectrum for a low-mass protostar. Previous interferometric studies (Bottinelli et al. 2004; Kuan et al. 2004) showed that the spectra toward the A component have broad lines (FWHMs up to $8 \mathrm{~km} \mathrm{~s}^{-1}$ ), whereas the lines toward the $\mathrm{B}$ component are much narrower (typically less than $2 \mathrm{~km} \mathrm{~s}^{-1}$ ), which makes it an ideal source for line identifications.

Actually, the rich spectrum of this source has made it possible to identify several COMs. Observations are reported for molecules containing up to four heavy atoms, such as ethyl
Table 2. Experimental spectroscopic parameters for the six observed conformers of 1,2-butanediol.

\begin{tabular}{|c|c|c|c|}
\hline & $\begin{array}{c}\mathrm{aG}^{\prime} \mathrm{Ag} \\
1-2 \\
\end{array}$ & $\begin{array}{c}\mathrm{g}^{\prime} \mathrm{G}^{\prime} \mathrm{Ag} \\
1-1 \\
\end{array}$ & $\begin{array}{c}\mathrm{gG}^{\prime} \mathrm{Aa} \\
1-3 \\
\end{array}$ \\
\hline$A / \mathrm{MHz}$ & $7830.1015(12)^{a}$ & $7821.8336(50)$ & $7694.37(13)$ \\
\hline$B / \mathrm{MHz}$ & $1945.15516(38)$ & $1930.6686(18)$ & $1937.5445(46)$ \\
\hline$C / \mathrm{MHz}$ & $1687.57121(38)$ & $1678.8135(20)$ & $1673.3921(45)$ \\
\hline$D_{J} / \mathrm{kHz}$ & $0.16080(41)$ & $0.1632(27)$ & $0.1558(21)$ \\
\hline$D_{J K} / \mathrm{kHz}$ & $1.9773(25)$ & $1.9263(15)$ & $1.8899(49)$ \\
\hline$D_{K} / \mathrm{kHz}$ & $8.253(16)$ & $7.80(12)$ & $-^{b}$ \\
\hline$d_{1} / \mathrm{Hz}$ & $-18.13(15)$ & $-17.30(39)$ & $-18.9(30)$ \\
\hline$d_{2} / \mathrm{Hz}$ & $-4.06(13)$ & $-3.26(34)$ & $2.72(83)$ \\
\hline$N^{c}$ & 77 & 192 & 70 \\
\hline$R M S^{d} / \mathrm{kHz}$ & 54 & 62 & 58 \\
\hline $\mathrm{a} / \mathrm{b} / \mathrm{c}^{e}$ & $y / y / y$ & $\mathrm{y} / \mathrm{y} / \mathrm{y}$ & $\mathrm{y} / \mathrm{n} / \mathrm{n}$ \\
\hline \multirow[t]{3}{*}{$\mu_{b} / \mu_{c}{ }^{f}$} & $2.1(4)$ & $1.1(1)$ & - \\
\hline & $\mathrm{aG}^{\prime} \mathrm{G}^{\prime} \mathrm{g}$ & $\mathrm{aG}^{\prime} \mathrm{Gg}$ & $\mathrm{g}^{\prime} \mathrm{GAa}$ \\
\hline & $2-3$ & $3-2$ & $4-2$ \\
\hline $\mathrm{A} / \mathrm{MHz}$ & $5509.3759(53)$ & $5966.8787(56)$ & $5961.4560(44)$ \\
\hline$B / \mathrm{MHz}$ & $2276.8059(36)$ & $2207.1497(25)$ & $2211.2757(20)$ \\
\hline$C / \mathrm{MHz}$ & $1739.5251(40)$ & $1936.0736(27)$ & $1937.8078(21)$ \\
\hline$D_{J} / \mathrm{kHz}$ & $0.4874(86)$ & $0.4438(49)$ & $0.4597(40)$ \\
\hline$D_{J K} / \mathrm{kHz}$ & $-0.508(75)$ & $0.386(28)$ & - \\
\hline$D_{K} / \mathrm{kHz}$ & $9.276(89)$ & $7.53(11)$ & $7.16(10)$ \\
\hline$d_{1} / \mathrm{Hz}$ & $-170.5(25)$ & $-57.60(95)$ & $-40.79(87)$ \\
\hline$d_{2} / \mathrm{Hz}$ & $-16.85(94)$ & $6.37(68)$ & $3.76(53)$ \\
\hline$H_{J K} / \mathrm{Hz}$ & $1.96(24)$ & - & - \\
\hline$H_{K} / \mathrm{Hz}$ & $2.19(45)$ & $-3.12(77)$ & - \\
\hline$N^{c}$ & 66 & 57 & 62 \\
\hline$R M S^{d} / \mathrm{kHz}$ & 51 & 52 & 72 \\
\hline $\mathrm{a} / \mathrm{b} / \mathrm{c}^{e}$ & $\mathrm{n} / \mathrm{y} / \mathrm{y}$ & $\mathrm{y} / \mathrm{y} / \mathrm{n}$ & $\mathrm{y} / \mathrm{y} / \mathrm{y}$ \\
\hline$\mu_{b} / \mu_{c}^{f}$ & $1.6(2)$ & - & $0.6(1)$ \\
\hline
\end{tabular}

Notes. ${ }^{(a)}$ Standard error in parentheses in the units of the last digit. ${ }^{(b)}$ Fixed to zero in the fit. ${ }^{(c)}$ Number of transitions. ${ }^{(d)}$ Root mean square deviation of the fit. ${ }^{(e)}$ Yes (y) or no (n) observation of $\mu_{a^{-}}, \mu_{b^{-}}$, and $\mu_{c^{-}}$ type transitions, respectively. ${ }^{(f)}$ Electric dipole moment components ratio estimated from the relative intensity of $\mu_{b}$-type and $\mu_{c}$-type transition quartets.

cyanide $\left(\mathrm{C}_{3} \mathrm{H}_{5} \mathrm{~N}\right)$, methyl formate, acetic acid (Cazaux et al. 2003) and glycoladehyde (Jørgensen et al. 2012; isomers with formula $\mathrm{C}_{2} \mathrm{H}_{4} \mathrm{O}_{2}$ ), acetone and propanal (isomers with formula $\left.\mathrm{C}_{3} \mathrm{H}_{6} \mathrm{O}\right)$, and even two conformers of ethylene glycol $\left(\mathrm{C}_{2} \mathrm{H}_{6} \mathrm{O}_{2}\right.$; Jørgensen et al. 2016).

Based on these considerations, IRAS 16293-2422 can be considered an adequate source for a search of 12BD. Several observations on this source were found in the public data of the ALMA Science Archive. Among these, project 2012.1.00712.S was selected because the frequency coverage (89.49-89.72, 92.78-93.01, 102.49-102.72, 103.18-103.41 GHz) and the spectral resolution $(60 \mathrm{kHz}$ channel spacing) are similar to those of our experiments.

The reduction of the data proceeded according to standard recipes exploiting the Common Astronomical Software Applications package (v4.2.1; McMullin et al. 2015). Assuming a better chance to find 12BD where the analog 12ED is present, the spectrum of the source was extracted within one $1^{\prime \prime} \times 1^{\prime \prime}$ synthesized beam size, centered on the $102.690 \mathrm{GHz}$ peak of 12ED in component $\mathrm{B}\left(\mathrm{RA}=16^{\mathrm{h}} 32^{\mathrm{m}} 22 .^{\mathrm{s}} 612\right.$; Dec $\left.=-24^{\circ} 28^{\prime} 32^{\prime \prime} .588\right)$. Subsequently, each spectral window was analyzed to identify the less crowded regions of the spectrum, best suited for the search of $12 \mathrm{BD}$. 


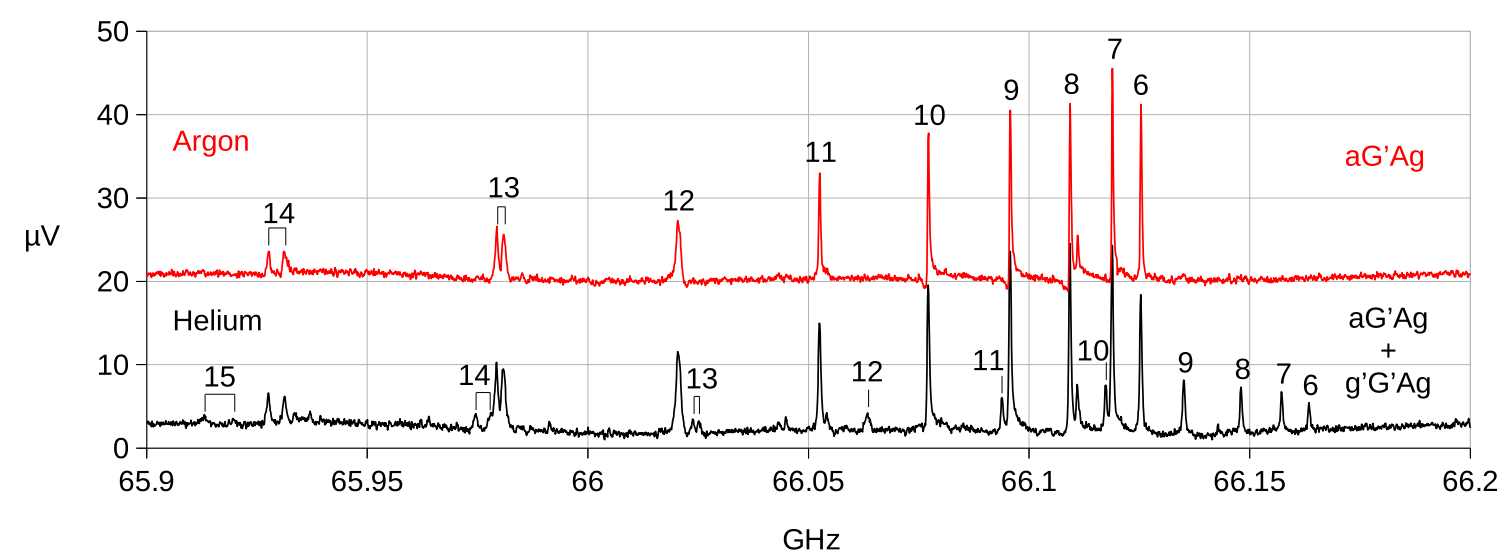

Fig. 4. Comparison between the 65.9-66.2 GHz spectra recorded using $\mathrm{Ar}$ (upper black red) and He (lower black trace) as carrier gas. In both spectra the $\mathrm{aG}^{\prime} \mathrm{Ag}$-12BD $K_{a}=6 \leftarrow 5 Q$-branch transitions can be recognized, whereas the same transitions of less stable $\mathrm{g}^{\prime} \mathrm{G}^{\prime} \mathrm{Ag}$ - $12 \mathrm{BD}$ are completely depleted in the Ar spectrum. Numbers above the peaks indicate the assigned $J$ quantum numbers.
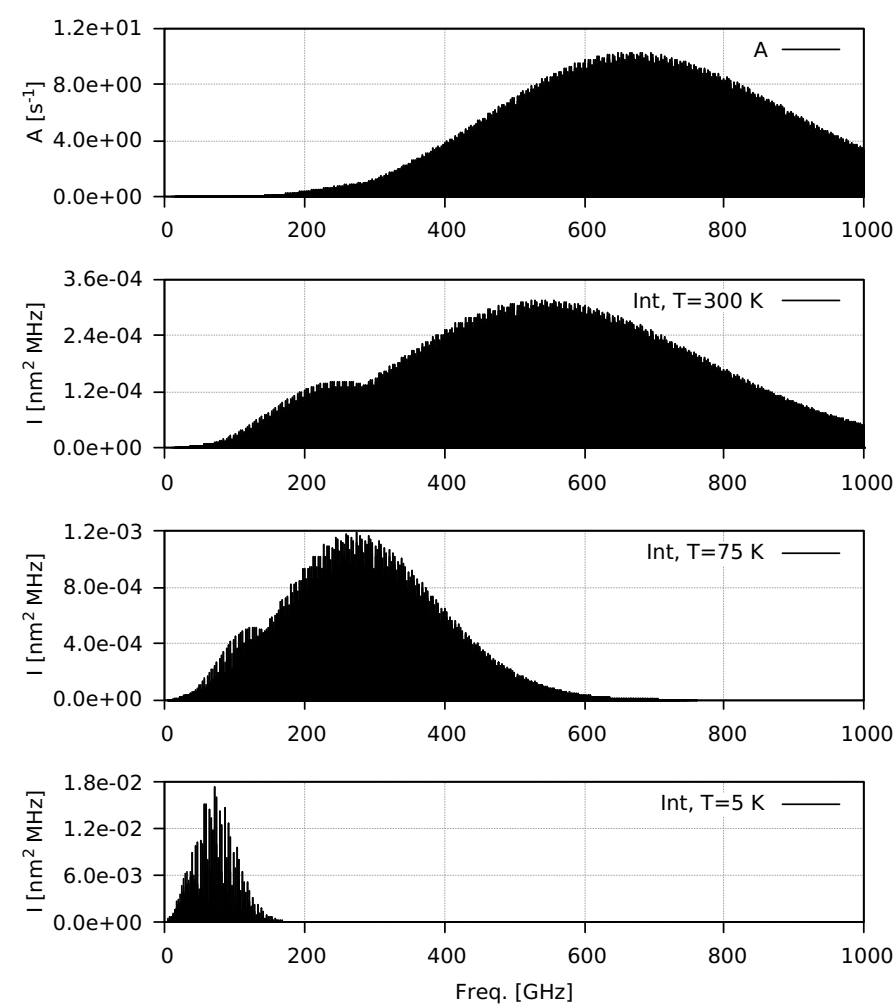

Fig. 5. Simulation of emission (top side) and absorption rotational spectra of $\mathrm{aG}^{\prime} \mathrm{Ag}-12 \mathrm{BD}$. Intensities are computed at 5, 75, and $300 \mathrm{~K}$.

Regarding the most stable conformer of $12 \mathrm{BD}$, the predicted transition lines in the available spectral windows are listed in Table A.2. Among these, a quartet of lines constituted by the $30_{x, 30}-29_{x, 29} \mu_{b}$ and $\mu_{c}$ R-type transitions appear promising to search. Indeed this quartet fulfills several requirements: $(i)$ the value of the predicted $A$ coefficient is high; (ii) the shape, constituted by four peaks in few $\mathrm{MHz}$, characterized by a specific pattern of separation is easily recognizable; (iii) the spectrum appears relatively free of contaminating lines; and (iv) the frequency range includes that spanned by the laboratory measurements.

The predicted lines are superimposed on the cleaned spectrum in Fig. 6. Two rotational transition lines belonging to methylformate $\left(\mathrm{CH}_{3} \mathrm{OCHO}\right)$ and methylacetylene $\left(\mathrm{CH}_{3} \mathrm{CCH}\right)$,
Table 3. Partition function of the six observed conformers of 1,2butanediol in the $5-300 \mathrm{~K}$ temperature range.

\begin{tabular}{rrrrrrr}
\hline \hline$T(\mathrm{~K})$ & $Q_{1-2}$ & $Q_{1-1}$ & $Q_{1-3}$ & $Q_{2-3}$ & $Q_{3-2}$ & $Q_{4-2}$ \\
\hline 300 & 172785 & 173976 & 175383 & 187529 & 173480 & 173319 \\
150 & 61089 & 61510 & 62007 & 66302 & 61334 & 61277 \\
75 & 21598 & 21747 & 21923 & 23441 & 21685 & 21665 \\
50 & 11757 & 11838 & 11933 & 12760 & 11804 & 11793 \\
25 & 4157 & 4185 & 4219 & 4511 & 4173 & 4169 \\
10 & 1052 & 1059 & 1067 & 1141 & 1056 & 1055 \\
5 & 372 & 374 & 377 & 403 & 373 & 373 \\
\hline
\end{tabular}

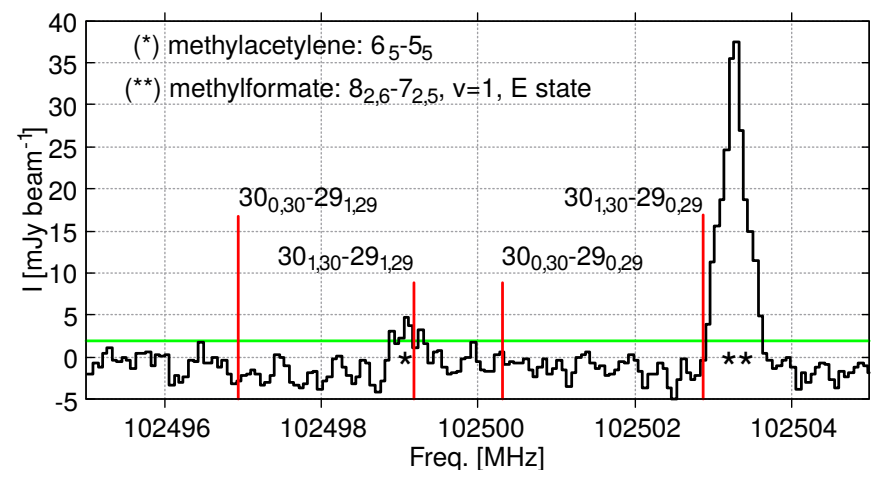

Fig. 6. Spectrum in the central beam toward the continuum peaks of IRAS 16293-2422 B. Predicted $30_{x, 30}-29_{x, 29}$ transition lines of $\mathrm{aG}^{\prime} \mathrm{Ag}-$ $12 \mathrm{BD}$ are overplotted as red sticks. The $X$-axis represents the frequencies in the rest frame of the system (i.e., corrected for the system $V_{\mathrm{LSR}}$ of $2.7 \mathrm{~km} \mathrm{~s}^{-1}$; Jørgensen et al. 2011). The green line is an indication of the rms level $\left(1.89 \mathrm{mJy}^{-1}\right.$ beam $\left.^{-1}\right)$ represented by a spectrum extracted from an off source position.

respectively, are clearly recognizable, while a lack of detection is observed for $12 \mathrm{BD}$.

We modeled the spectrum extracted from the ALMA data using MCWeeds (Giannetti et al. 2017) to obtain an upper limit for the 12BD column density. Assuming a temperature of $300 \mathrm{~K}$ and a line width of $1 \mathrm{~km} \mathrm{~s}^{-1}$, as measured for ethylene glycol (Jørgensen et al. 2016), we estimate $N<1 \times 10^{13} \mathrm{~cm}^{-2}(95 \%$ credible interval).

However, searching of 12BD features could be successful at higher frequency spectral ranges. In fact, considering a temperature of the source around $300 \mathrm{~K}$ (Jørgensen et al. 2011) the most 
populated rotational levels with the highest possible Einstein $A$ coefficients lie in the $400-800 \mathrm{GHz}$ frequency range (Fig. 5), corresponding to bands $8-9$.

Searches for upper bands in available databases have not been performed yet, since additional frequency laboratory measurements are needed to fix the higher order centrifugal distortion constants required for reliable spectral predictions.

\section{Conclusions}

In this work, features of millimetre rotational spectroscopy coupled to supersonic expansion have been exploited to analyze, for the first time, the spectrum of 12BD. Actually, at room temperature, the conformational population of this highly flexible diol is distributed on a large numbers of conformations practically preventing the assignment. The spectra recorded in the 59.6103.6 GHz frequency range have allowed the unequivocal identification of six different species, determining that the $\mathrm{aG}^{\prime} \mathrm{Ag}$ conformer is that with the minimum energy. Besides the rotational constants, centrifugal distortion constants were also determined: in particular, four quartic constants for $\mathrm{gG}^{\prime} \mathrm{Aa}$ and $\mathrm{g}^{\prime} \mathrm{GAa}$, the full quartic set for $\mathrm{aG}^{\prime} \mathrm{Ag}$ and $\mathrm{g}^{\prime} \mathrm{G}^{\prime} \mathrm{Ag}$, and additional sextic contributions for $\mathrm{aG}^{\prime} \mathrm{Gg}$ and $\mathrm{aG}^{\prime} \mathrm{G}^{\prime} \mathrm{g}$.

The experimental spectroscopic constants and theoretical electric dipole moment components were used to predict with high precision the rotational spectrum of each of the six observed conformers up to $163 \mathrm{GHz}$ based on the measurements done. Selected lines of the global minimum aG'Ag-12BD were searched toward the IRAS 16293-2422 B source in the band 3 observations of the ALMA project 2012.1.00712.S. No detection was found, but this could be justified by the fact that the maximum spectral signal of this kind of heavy compounds is predicted at higher frequencies (bands 8-9). The ALMA project, from which the data were extracted, was in fact, not specifically aimed at searching this molecule. Our predictions may allow now us to define observational ALMA campaigns dedicated to 12BD.

Hence, on the basis of our predictions, additional laboratory measurements at submillimeter wavelengths can be performed and new specific observations in COMs-reach sources could be attempted.

Acknowledgements. This paper makes use of the following ALMA data ADS/JAO.ALMA\#2012.1.00712.S. ALMA is a partnership of ESO (representing its member states), NSF (USA) and NINS (Japan), together with NRC (Canada), MOST and ASIAA (Taiwan), and KASI (Republic of Korea), in cooperation with the Republic of Chile. The Joint ALMA Observatory is operated by ESO, AUI/NRAO, and NAOJ. We wish to thank Fabrizio Villa and Francesco Cuttaia of Cryowave Laboratory, INAF-OAS, via Gobetti 101, Bologna for sharing the frequency multiplier equipment. We acknowledge the CINECA award under the ISCRA initiative and the Italian ALMA Regional Center for the availability of high performance computing resources and support. CC acknowledges the Spanish Government for a "Juan de la Cierva" contract. These investigation have been supported by the Italian MIUR (Attività Base di Ricerca funds and PRIN 2015 F59J3R 004 PE4) and the University of Bologna (Ricerca Fondamentale Orientata funds).

\section{References}

Biver, N., Bockelée-Morvan, D., Debout, V., et al. 2014, A\&A, 566, L5 Bossa, J. B., Ordu, M. H., Müller, H. S. P., Lewen, F., \& Schlemmer, S. 2014 A\&A, 570, A12
Bottinelli, S., Ceccarelli, C., Neri, R., et al. 2004, ApJ, 617, L69 Brouillet, N., Despois, D., Lu, X.-H., et al. 2015, A\&A, 576, A129

Brünken, S., \& Schlemmer, S. 2015, in Conditions and Impact of Star Formation, eds R., Simon, R., Schaaf, \& J., Stutzki, EAS Pub. Ser., 75, 295

Calabrese, C., Vigorito, A., Maris, A., et al. 2015, J. Phys. Chem. A, 119, 11674

Caminati, W. 1981, J. Mol. Spectr., 86, 193

Caminati, W., \& Corbelli, G. 1982, J. Mol. Struct., 78, 197

Caminati, W., Melandri, S., \& Favero, P. G. 1995, J. Mol. Spectr., 171, 394

Cazaux, S., Tielens, A. G. G. M., Ceccarelli, C., et al. 2003, ApJ, 593, L51

CDMS 2001, The Cologne Database for Molecular Spectroscopy, https:// www .astro.uni-koeln. de/cdms/molecules

Christen, D., \& Müller, H. S. P. 2003, Phys. Chem. Chem. Phys., 5, 3600

Christen, D., Coudert, L. H., Suenram, R. D., \& Lovas, F. J. 1995, J. Mol. Spectr., 172, 57

Christen, D., Coudert, L. H., Larsson, J. A., \& Cremer, D. 2001, J. Mol. Spectr., 205,185

Coutens, A., Persson, M. V., Jørgensen, J. K., Wampfler, S. F., \& Lykke, J. M. 2015, A\&A, 576, A5

Crovisier, J., Bockelée-Morvan, D., Biver, N., et al. 2004, A\&A, 418, L35

Evangelisti, L., Gou, Q., Spada, L., Feng, G., \& Caminati, W. 2013, Chem. Phys. Letters, 556, 55

Favre, C., Pagani, L., Goldsmith, P., et al. 2017, A\&A, 604, L2

Frisch, M. J., Trucks, G. W., Schlegel, H. B., et al. 2013, Gaussian 09, Revision D.01 (Wallingford, CT: Gaussian Inc.)

Fuente, A., Cernicharo, J., Caselli, P., et al. 2014, A\&A, 568, A65

Giannetti, A., Leurini, S., Wyrowsky, F., et al. 2017, A\&A, 603, A33

Grimme, S., Antony, J., Ehrlich, S., \& Krieg, H. 2010, J. Chem. Phys, 132, 154104

Hollis, J. M., Lovas, F. J., Jewell, P. R., \& Coudert, L. H. 2002, ApJ, 571, L59

Jørgensen, J. K., Bourke, T. L., Nguyen Luong, Q., \& Takakuva, S. 2011, A\&A, 534, A100

Jørgensen, J. K., Favre, C., Bisschop, S. E., et al. 2012, ApJ, 757, L4

Jørgensen, J. K., van der Wiel, M. H. D., Coutens, A., et al. 2016, A\&A, 595, A117

Kalenskii, S. V., \& Johansson, L. E. B. 2010, Astron. Rep., 54, 1084

Kuan, Y.-J., Huang, H.-C., Charnley, S. B., et al. 2004, ApJ, 616, L27

Lockley, J. L., Hearn, J. P. I., King, A. K., \& H.B, J. 2002, J. Mol. Struct., 612 199

Lovas, F. J., Plusquellic, D. F., Pate, B. H., et al. 2009, J. Mol. Spectr., 257, 82

Lykke, J. M., Favre, C., Bergin, E. A., \& Jørgensen, J. K. 2015, A\&A, 757, L4

Marstokk, K.-M., \& Møllendal, H. 1974, J. Mol. Struct., 22, 301

Maury, A. J., Belloche, A., André, P., et al. 2014, A\&A, 563, L2

McMullin, J. P., Waters, B., Schiebel, D., Young, W., \& Golap, K. 2015, in Astronomical Data Analysis Software and Systems XVI, eds. R. A. Shaw, F. Hill, \& D. J. Bell, ASP Conf. Ser., 376, 127

Melandri, S., Caminati, W., Favero, L. B., Millemaggi, A., \& Favero, P. G. 1995 J. Mol. Struct., 352/353, 253

Melandri, S., Maccaferri, G., Maris, A., et al. 1996, Chem. Phys. Lett., 261, 267

Müller, H. S. P., \& Christen, D. 2004, J. Mol. Spectr., 228, 298

Müller, H. S. P., Thorwirth, S., Roth, D. A., \& Winnewisser, G. 2001, A\&A, 370, L49

Müller, H. S. P., Schlöder, F., Stutzki, J., \& Winnewisser, G. 2005, J. Mol, Struct., 742,215

Paul, J., Hearn, I., \& Howard, B. J. 2007, Mol. Phys., 105, 825

Pickett, H. M. 1991, J. Mol. Spectr., 148, 371

Plusquellic, D. F., Lovas, F. J., Pate, B. H., et al. 2009, J. Phys. Chem. A, 113, 12911

PRIMOS 2013, The Prebiotic Interstellar Molecule Survey Legacy Project, http://www.cv.nrao.edu/aremijan/PRIMOS

Remijan, A. J., Hollis, J. M., Jewell, P. R., Lovas, F., \& Corby, J. 2013, in AAS Meeting Abstracts, 221, 352.08

Requena-Torres, M. A., Martín-Pintado, J., Martín, S., \& Morris, M. R. 2008, ApJ, 672, 352

Ruoff, R. S., Klots, T. D., Emilson, T., \& Gutowski, H. S. 1990, J. Chem. Phys., 93, 3142

Steele, W. V., Chirico, R. D., Knipmeyer, S. E., \& Nguyen, A. 1996, J. Chem. Eng. Data, 41, 1255

Velino, B., Favero, L. B., Maris, A., \& Caminati, W. 2011, J. Phys. Chem. A, 115,9585

Verevkin, S. P. 2004, Fluid Phase Equil., 224, 23

Walder, E., Bauder, A., \& Günthard, H. 1980, Chem. Phys., 51, 223

Watson, J. K. G. 1977, Vibrational Spectra and Structure (New York/Amsterdam: Elsevier) 


\section{Appendix A: Supplementary material}

The newly recorded experimental transition frequencies for the six observed conformers of 1,2-butanediol are available at the CDS. Only the first 30 lines measured for the $\mathrm{aG}^{\prime} \mathrm{Ag}$ conformer of 1,2-butanediol appear here (Table A.1). The table gives the rotational quantum numbers $J, K_{a}$, and $K_{c}$ for the upper state followed by those for the lower state. The observed transition frequency is given in megahertz units with the residual between observed frequency and that calculated from the final set of spectroscopic parameters and its uncertainty.
In addition also the predicted rotational spectra in the $0-163 \mathrm{GHz}$ frequency range are also available at the CDS. Only a portion relative to the $\mathrm{aG}^{\prime} \mathrm{Ag}$ conformer is given below (Table A.2). The data listing includes the following: the predicted rest frequencies and $1 \sigma$ uncertainties, the upper state degeneracy and energy, the line strength factors $S_{i j} \mu_{g}^{2}$, and the Einstein's $A$ coefficients for spontaneous emission. The prediction sample comprises lines, which have upper state energy $E_{\text {up }}<100 \mathrm{~K}$, and Einstein's coefficient $A>10^{-7} \mathrm{~s}^{-1}$.

Table A.1. Selected measured rest frequencies, residuals between observed frequency and that calculated from the final set of spectroscopic parameters and experimental uncertainties for the ground vibrational state of the $\mathrm{aG}^{\prime} \mathrm{Ag}$ (1-2) conformer of 1,2-butanediol.

\begin{tabular}{|c|c|c|c|c|c|c|c|c|c|}
\hline (1) & $J^{\prime}$ & $\begin{array}{l}K_{a}^{\prime} \\
(3) \\
\end{array}$ & $\begin{array}{l}K_{c}^{\prime} \\
(4) \\
\end{array}$ & (5) & $\begin{array}{l}K_{a} \\
(6) \\
\end{array}$ & $\begin{array}{l}K_{c} \\
(7) \\
\end{array}$ & $\begin{array}{c}\text { Rest freq. } \\
(\mathrm{MHz}) \\
(8)\end{array}$ & $\begin{array}{c}\mathrm{O}-\mathrm{C} \\
(\mathrm{MHz}) \\
(9)\end{array}$ & $\begin{array}{c}\text { Unc. } \\
(\mathrm{MHz}) \\
(10)\end{array}$ \\
\hline 1 & 16 & 2 & 14 & 15 & 2 & 13 & 59930.23 & 0.01 & 0.05 \\
\hline 2 & 20 & 2 & 18 & 19 & 3 & 17 & 59976.02 & 0.00 & 0.05 \\
\hline 3 & 10 & 2 & 9 & 9 & 1 & 9 & 60144.66 & 0.00 & 0.05 \\
\hline 4 & 12 & 2 & 10 & 11 & 1 & 10 & 60205.72 & -0.02 & 0.05 \\
\hline 5 & 11 & 3 & 8 & 11 & 0 & 11 & 60207.34 & 0.01 & 0.05 \\
\hline 6 & 11 & 3 & 8 & 11 & 0 & 11 & 60207.34 & 0.01 & 0.05 \\
\hline 7 & 5 & 4 & 1 & 4 & 3 & 2 & 60261.44 & 0.06 & 0.05 \\
\hline 8 & 5 & 4 & 2 & 4 & 3 & 2 & 60261.44 & 0.09 & 0.05 \\
\hline 9 & 9 & 2 & 7 & 8 & 0 & 8 & 60506.70 & -0.02 & 0.05 \\
\hline 10 & 15 & 2 & 14 & 14 & 1 & 13 & 60646.78 & 0.01 & 0.05 \\
\hline 11 & 17 & 2 & 16 & 16 & 2 & 15 & 60737.95 & 0.00 & 0.05 \\
\hline 12 & 18 & 1 & 17 & 17 & 2 & 16 & 61051.59 & 0.02 & 0.05 \\
\hline 13 & 17 & 1 & 16 & 16 & 1 & 15 & 61624.47 & 0.02 & 0.05 \\
\hline 14 & 9 & 3 & 7 & 8 & 2 & 6 & 61680.90 & -0.01 & 0.05 \\
\hline 15 & 18 & 0 & 18 & 17 & 1 & 17 & 61817.80 & -0.01 & 0.05 \\
\hline 16 & 9 & 3 & 6 & 8 & 2 & 6 & 61831.62 & 0.00 & 0.05 \\
\hline 17 & 17 & 10 & 8 & 16 & 10 & 7 & 61838.33 & -0.04 & 0.05 \\
\hline 18 & 17 & 10 & 7 & 16 & 10 & 6 & 61838.33 & -0.04 & 0.05 \\
\hline 19 & 17 & 9 & 9 & 16 & 9 & 8 & 61855.90 & -0.03 & 0.05 \\
\hline 20 & 17 & 9 & 8 & 16 & 9 & 7 & 61855.90 & -0.03 & 0.05 \\
\hline 21 & 17 & 8 & 10 & 16 & 8 & 9 & 61879.95 & -0.04 & 0.05 \\
\hline 22 & 17 & 8 & 9 & 16 & 8 & 8 & 61879.95 & -0.04 & 0.05 \\
\hline 23 & 17 & 7 & 11 & 16 & 7 & 10 & 61914.86 & 0.07 & 0.05 \\
\hline 24 & 17 & 7 & 10 & 16 & 7 & 9 & 61914.86 & 0.06 & 0.05 \\
\hline 25 & 17 & 3 & 15 & 16 & 3 & 14 & 61918.42 & 0.05 & 0.05 \\
\hline 26 & 17 & 6 & 12 & 16 & 6 & 11 & 61968.64 & 0.14 & 0.05 \\
\hline 27 & 17 & 6 & 11 & 16 & 6 & 10 & 61968.64 & -0.26 & 0.05 \\
\hline 28 & 18 & 1 & 18 & 17 & 1 & 17 & 61984.63 & -0.03 & 0.05 \\
\hline 29 & 18 & 0 & 18 & 17 & 0 & 17 & 62048.18 & 0.04 & 0.05 \\
\hline 30 & 17 & 5 & 13 & 16 & 5 & 12 & 62054.77 & 0.03 & 0.05 \\
\hline
\end{tabular}

Notes. (1) Listing number n. (2-4) Upper state rotational quantum numbers $J_{K a^{\prime}, K c^{\prime}}$. (5-7) Lower state rotational quantum numbers $J_{K a, K c}$. (8) Observed transition frequency. (9) Residual between observed frequency and that calculated from the final set of spectroscopic parameters O-C. (10) Experimental uncertainty Unc. The full Table A.1 is available at the CDS. 
Table A.2. Selected predicted rest frequencies, estimated accuracies, and lines strengths for the ground vibrational state of the aG' $\mathrm{Ag}(1-2)$ conformer of 1,2-butanediol.

\begin{tabular}{|c|c|c|c|c|c|c|c|c|c|c|c|}
\hline$J^{\prime}$ & $\begin{array}{l}K_{a}^{\prime} \\
(2)\end{array}$ & $\begin{array}{l}K_{c}^{\prime} \\
(3)\end{array}$ & (4) & $\begin{array}{l}K_{a} \\
(5)\end{array}$ & $\begin{array}{l}K_{c} \\
(6)\end{array}$ & $\begin{array}{l}\text { Rest freq. } \\
(\mathrm{MHz}) \\
(7)\end{array}$ & $\begin{array}{c}\text { Error } \\
(\mathrm{MHz}) \\
(8)\end{array}$ & $\begin{array}{r}G_{\text {up }} \\
(9)\end{array}$ & $\begin{array}{l}E_{\text {up }} \\
(\mathrm{K}) \\
(10)\end{array}$ & $\begin{array}{l}S \mu_{g}^{2} \\
\left(\mathrm{D}^{2}\right) \\
(11)\end{array}$ & $\begin{array}{c}A \\
\left(\mathrm{~s}^{-1}\right) \\
(12)\end{array}$ \\
\hline 32 & 4 & 28 & 32 & 3 & 30 & 89492.71 & 0.27 & 65 & 98.520 & $3.889 \mathrm{E}-01$ & $3.245 \mathrm{E}-06$ \\
\hline 17 & 6 & 12 & 18 & 3 & 15 & 89506.84 & 0.02 & 35 & 37.117 & $1.586 \mathrm{E}-02$ & $1.324 \mathrm{E}-07$ \\
\hline 24 & 8 & 17 & 24 & 7 & 17 & 89533.89 & 0.02 & 49 & 70.881 & $1.991 \mathrm{E}+00$ & $1.664 \mathrm{E}-05$ \\
\hline 24 & 8 & 16 & 24 & 7 & 17 & 89533.95 & 0.02 & 49 & 70.881 & $4.081 \mathrm{E}+01$ & $3.410 \mathrm{E}-04$ \\
\hline 18 & 5 & 14 & 18 & 3 & 15 & 89534.42 & 0.02 & 37 & 37.118 & $3.602 \mathrm{E}-02$ & $3.010 \mathrm{E}-07$ \\
\hline 24 & 8 & 17 & 24 & 7 & 18 & 89535.92 & 0.02 & 49 & 70.881 & $4.081 \mathrm{E}+01$ & $3.410 \mathrm{E}-04$ \\
\hline 24 & 8 & 16 & 24 & 7 & 18 & 89535.98 & 0.02 & 49 & 70.881 & $1.991 \mathrm{E}+00$ & $1.664 \mathrm{E}-05$ \\
\hline 21 & 4 & 17 & 21 & 2 & 20 & 89584.59 & 0.04 & 43 & 45.211 & $3.765 \mathrm{E}-02$ & $3.151 \mathrm{E}-07$ \\
\hline 28 & 7 & 21 & 29 & 4 & 26 & 89589.38 & 0.09 & 57 & 85.174 & $8.523 \mathrm{E}-02$ & $7.135 \mathrm{E}-07$ \\
\hline 23 & 8 & 16 & 23 & 7 & 16 & 89632.77 & 0.02 & 47 & 66.681 & $1.902 \mathrm{E}+00$ & $1.595 \mathrm{E}-05$ \\
\hline 23 & 8 & 15 & 23 & 7 & 16 & 89632.80 & 0.02 & 47 & 66.681 & $3.863 \mathrm{E}+01$ & $3.238 \mathrm{E}-04$ \\
\hline 23 & 8 & 16 & 23 & 7 & 17 & 89633.89 & 0.02 & 47 & 66.681 & $3.863 \mathrm{E}+01$ & $3.238 \mathrm{E}-04$ \\
\hline 23 & 8 & 15 & 23 & 7 & 17 & 89633.92 & 0.02 & 47 & 66.681 & $1.902 \mathrm{E}+00$ & $1.595 \mathrm{E}-05$ \\
\hline 22 & 8 & 15 & 22 & 7 & 15 & 89719.41 & 0.02 & 45 & 62.658 & $1.811 \mathrm{E}+00$ & $1.523 \mathrm{E}-05$ \\
\hline 22 & 8 & 14 & 22 & 7 & 15 & 89719.42 & 0.02 & 45 & 62.658 & $3.645 \mathrm{E}+01$ & $3.065 \mathrm{E}-04$ \\
\hline 22 & 8 & 15 & 22 & 7 & 16 & 89720.01 & 0.02 & 45 & 62.658 & $3.645 \mathrm{E}+01$ & $3.065 \mathrm{E}-04$ \\
\hline 22 & 8 & 14 & 22 & 7 & 16 & 89720.02 & 0.02 & 45 & 62.658 & $1.811 \mathrm{E}+00$ & $1.523 \mathrm{E}-05$ \\
\hline 18 & 6 & 12 & 19 & 3 & 17 & 92767.31 & 0.03 & 37 & 40.267 & $1.926 \mathrm{E}-02$ & $1.790 \mathrm{E}-07$ \\
\hline 27 & 7 & 20 & 28 & 4 & 25 & 92974.40 & 0.07 & 55 & 80.261 & $7.105 \mathrm{E}-02$ & $6.648 \mathrm{E}-07$ \\
\hline 30 & 0 & 30 & 29 & 1 & 29 & 102496.62 & 0.03 & 61 & 77.081 & $9.391 \mathrm{E}+01$ & $1.177 \mathrm{E}-03$ \\
\hline 12 & 4 & 9 & 12 & 1 & 12 & 102496.95 & 0.02 & 25 & 18.248 & $3.639 \mathrm{E}-02$ & $4.563 \mathrm{E}-07$ \\
\hline 30 & 1 & 30 & 29 & 1 & 29 & 102499.18 & 0.03 & 61 & 77.081 & $4.899 \mathrm{E}+01$ & $6.142 \mathrm{E}-04$ \\
\hline 30 & 0 & 30 & 29 & 0 & 29 & 102500.31 & 0.03 & 61 & 77.080 & $4.899 \mathrm{E}+01$ & $6.142 \mathrm{E}-04$ \\
\hline 30 & 1 & 30 & 29 & 0 & 29 & 102502.87 & 0.03 & 61 & 77.081 & $9.392 \mathrm{E}+01$ & $1.178 \mathrm{E}-03$ \\
\hline 28 & 6 & 23 & 27 & 6 & 22 & 102591.50 & 0.02 & 57 & 81.506 & $4.376 \mathrm{E}+01$ & $5.502 \mathrm{E}-04$ \\
\hline 28 & 6 & 22 & 27 & 6 & 21 & 102688.25 & 0.02 & 57 & 81.520 & $4.377 \mathrm{E}+01$ & $5.518 \mathrm{E}-04$ \\
\hline 28 & 5 & 24 & 27 & 5 & 23 & 102719.83 & 0.02 & 57 & 78.429 & $4.440 \mathrm{E}+01$ & $5.602 \mathrm{E}-04$ \\
\hline 18 & 4 & 15 & 17 & 3 & 14 & 103260.20 & 0.01 & 37 & 34.554 & $1.807 \mathrm{E}+01$ & $2.316 \mathrm{E}-04$ \\
\hline 24 & 3 & 22 & 24 & 0 & 24 & 103267.79 & 0.10 & 49 & 54.948 & $4.944 \mathrm{E}-02$ & $6.338 \mathrm{E}-07$ \\
\hline
\end{tabular}

Notes. (1-3) Upper state rotational quantum numbers $J^{\prime}{ }_{K a^{\prime}, K c^{\prime}}$. (4-6) Lower state rotational quantum numbers $J_{K a, K c}$. (7) Rest frequency evaluated from the spectroscopic constants. (8) Estimated error of the prediction at $1 \sigma$ level. (9) Upper state degeneracy. (10) Upper state energy. (11) Transition strength $S \mu_{g}^{2}(g=a, b, c$ theoretical electric dipole moment component). (12) Einstein's $A$ coefficient. The full Table A.2 is available at the CDS. 